\title{
IGF-1 on Titanium Alloy Implants Enhances Their Osseointegration in the Rat Femur: A SEM study
}

\author{
G. Sovak ${ }^{1}$, A.Weiss ${ }^{1}$, I. Gotman ${ }^{2}$ \\ ${ }^{1}$ Department of Anatomy \& Cell Biology, The Rappaport Faculty of Medicine, Technion_Israel \\ Institute of Technology, P.O.Box 9649, Haifa 31096, Israel \\ ${ }^{2}$ Department of Materials Engineering, Technion, Haifa 31096, Israel
}

Biological fixation of uncemented endosseous implants requires the apposition of bone onto implant's surface in a process named osseointegration [1]. Osseointegration progresses slowly, and requires long periods of unloading. We have previously reported that growth hormone $(\mathrm{GH})$ enhances $\mathrm{Ti}$ alloy implant osseointegration in adult rats [2]. GH is known to exert both direct effects on bone, as well as indirect effects, by stimulating hepatic and local production of IGF-1 [3]. IGF-1 is expensive and unsafe for systemic administration. The aim of the present research was to study the direct effects of IGF-1 on titanium alloy implants fixation, by implanting implants preloaded with IGF-1.

Ti-6Al-4V pins were were presoaked for $24 \mathrm{hrs}$ in $0.1 \mathrm{mg} / \mathrm{ml}$ of human recombinant IGF-1 solution and then implanted in the distal femurs of 6-month-old Wistar female rats. IGF-1 adsorption to the implants was verified by ELISA. Animals were euthanized after 10 days. To evaluate mineralization, animals received $30 \mathrm{mg} / \mathrm{kg}$ body weight of oxytetracycline, $72 \mathrm{hrs}$ before sacrifice. Undecalcified sections of the femurs with implants were examined by scanning electron microscope (SEM). Morphometric analyses were performed with an aid of ImagePro software, as described previously [4]. SEM revealed that the implants underwent fixation in bone within the distal epiphyses; in the diaphyses only bone marrow was found around the control implantsin the medullary cavity, while the JGF-1 loaded pins were surrounded by trabecular bone (Fig. 1A-D). Two parameters of osseointegration: bone volume (BV) and bone-implant contact (BIC) were measured on SEM images of cross-sections from femoral epiphyses and diaphyses. Bone volume was measured within a distance of $0.3 \mathrm{~mm}$ from implants' surface. The morphometric results indicate that both the BV and BIC were significantly increased in increased around the IGF-1 pins in comparison to the controls (Table 1). Also, tetracycline fluorescence revealed an increase in mineralization around JGF-1 loaded pins in comparison to control pins.

In conclusion, our findings revealed that adsorption of IGF-1 directly onto the implant's surface improves implant fixation without unnecessary systemic effects of IGF-1.

\section{References}

[1] P.I. Branemark et al., Quintessence (1985) 11.

[2] Y. Segev et al. M\&M 2010.

[3] E. Canalis, Calcif. Tiss. Int. 53 (suppl.) (1993) S90.

[4] G. Sovak et al., J. Bone Joint Surg. [Br]; 82-B (2000) 290. 

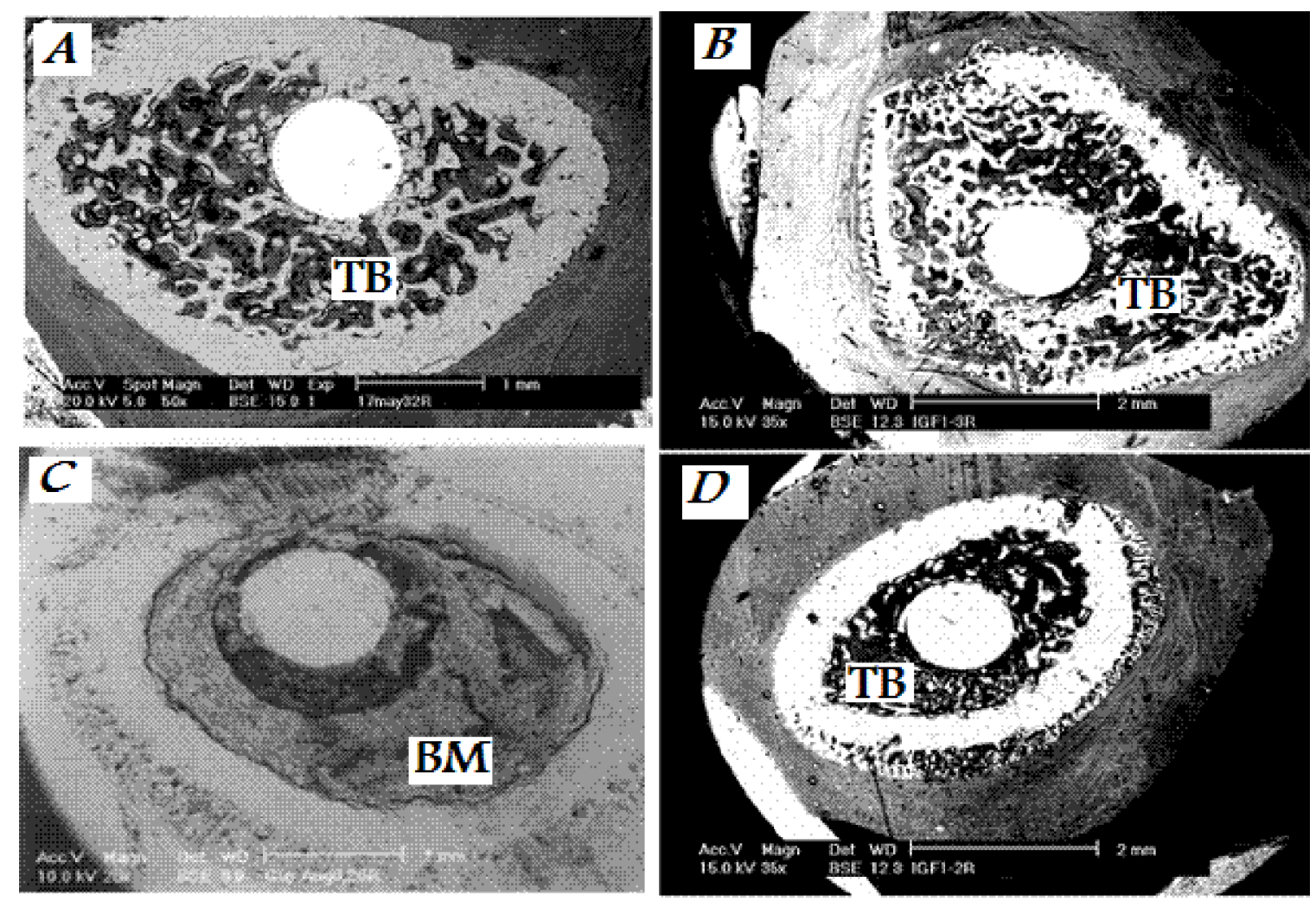

Fig. 1. SEM images of transverse sections of rat femurs, 10 days after implantation. A,epiphysiscontrol implant; B, epiphysis - IGF-1 implant; C, diaphysis-control implant; D, diaphysis - IGF-1 implant. BM, bone marrow; TB, trabecular bone. Note that no TB is seen in the medullary cavity of the femur with control implant (C).

Table 1. Effect of GH bone volume and bone-implant contact in the rat femur.

\begin{tabular}{|c|c|c|c|c|c|}
\hline Site & Implant & Bone volume $(\%)$ & $\mathrm{p}<$ & Bone-implant contact $(\%$ & $\mathrm{p}<$ \\
\hline Epiphysis & $\begin{array}{l}\text { Control } \\
\text { IGF-1 }\end{array}$ & $\begin{array}{l}47.4 \pm 4.3 \\
48.8 \pm 8.6\end{array}$ & $\begin{array}{c}- \\
\mathrm{NS}\end{array}$ & $\begin{array}{l}72.5 \pm 7.9 \\
65.8 \pm 7.2\end{array}$ & $\begin{array}{c}- \\
\mathrm{NS}\end{array}$ \\
\hline Diaphysis & $\begin{array}{l}\text { Control } \\
\text { IGF-1 }\end{array}$ & $\begin{array}{l}0 \\
24.8 \pm 7.6\end{array}$ & - & $\begin{array}{l}4.3 \pm 1.1 \\
37.1 \pm 14\end{array}$ & - \\
\hline
\end{tabular}

$\mathrm{BV}$ and BIC were measured on SEM images with ImagePro. p-significance in comparison to control by ANOVA; NS-nonsignificant. 